\title{
Gerencia Social: un análisis bibliométrico desde Dimensions (1989-2020)
}

\author{
Social Management: a bibliometric analysis from Dimensions (1989-2020)
}

\author{
Juana Patricio Hidalgo Euribe, ${ }^{\text {a }}$ Cesar H. Limaymanta, ${ }^{2}$ Armando Edison Velazco Ríos, ${ }^{3}$ \\ Salvador E. Vasquez-Moctezuma ${ }^{4}$ \\ ORCID ID: https://orcid.org/0000-0002-0918-338X \\ (iD) ORCID ID: $\underline{\text { https://orcid.org/0000-0002-8797-4275 }}{ }^{2}$ \\ (iD) ORCID ID: https://orcid.org/0000-0002-2480-4308 ${ }^{3}$ \\ (iD) ORCID ID: https://orcid.org/0000-0002-7300-784X \\ Universidad Nacional Mayor de San Marcos, Lima, Perú ${ }^{123}$ \\ Universidad Nacional Autónoma de México, Coyoacán, México DF ${ }^{4}$
}

Recibido: 04 agosto de 2021

Aceptado: 09 de diciembre de 2021

\section{Resumen}

El propósito de la presente investigación es analizar, desde una perspectiva latinoamericana, la producción científica referida a la gerencia social en la base de datos Dimensions entre los años 1989 y 2020 . Desde una metodología bibliométrica con enfoque cuantitativo-descriptivo, el estudio busca llenar un vacío de conocimiento sobre las características de las publicaciones sobre gerencia social. Se presentan indicadores de producción, la ley de Bradford, el mapeo de redes bibliométricas según coautoría y coocurrencia de palabras clave. Los resultados muestran el carácter multidimensional de la gerencia social como campo disciplinar. Se evidencia también una creciente producción y concentración de publicaciones en algunos países de la región latinoamericana. Además, se identifican autores, categorías temáticas y revistas de mayor producción. En este caso, Brasil es el país que destaca en cuanto a la producción científica que publican sobre esta temática.

Palabras clave: gerencia social, bibliometría, políticas sociales, base de datos Dimensions, estado del arte

\begin{abstract}
The objective of this study is to describe and analyze, from a Latin American perspective, the scientific production that belongs to Social Management in the Dimensions database, between 1989 and 2020. Using a bibliometric methodology and a quantitative-descriptive scope, it aims to fill a gap in knowledge referred to the evolution of the research topics in Social Management. Productivity indicators and mapping of bibliometric networks by co-authors and keywords are
\end{abstract}

${ }^{a}$ Correspondencia al autor

E-mail: jhidalgoe@unmsm.edu.pe 
shown. Results show the multidimensional character of Social Management as an academic field. We present evidence of the growing production and concentration of publications in some countries of the Latin-American region. We also show the most productive authors, thematic categories, and journals. In this case, the country that stands out the most in terms of scientific production, number of authors and journals is Brazil.

Keywords: Social Management, bibliometrics, social policy, Dimensions database, state of the art

\section{Introducción}

La noción de gerencia social tuvo un desarrollo propio en el ámbito latinoamericano en la década de los noventa (Tenorio, 1997; Cançado et al., 2016; De Oliveira Menon \& De Souza Coelho, 2019; Tenorio \& Teixeira, 2021). En ese contexto socioeconómico crítico, se buscó el abordaje de las políticas públicas, desde la perspectiva del gerenciamiento y la gestión, en especial de las políticas sociales. Se configuró en sus inicios como aquel campo de conocimiento cuyo fin era la reducción de la pobreza, el fortalecimiento de la democracia y la ciudadanía, mediante el desarrollo de procesos participativos que generen valor público y que se ubica entre las áreas de desarrollo social, las políticas públicas y la gerencia pública (Instituto Interamericano para el Desarrollo Económico y Social [INDES], 2006). A la vez, surge en paralelo a la nueva concepción alrededor de la gestión pública o nueva gestión pública. Así mismo, en el plano empresarial se daban cambios como respuesta a las nuevas exigencias del ámbito privado frente a los cuales la gerencia social se configura como una salida a la ortodoxia positivista y la racionalidad utilitaria propias del pensamiento administrativo tradicional (Cançado et al., 2011).

La literatura referida a esta disciplina se desplegó principalmente en sus primeros años bajo el término de gerencia social (Tenorio \& Teixeira, 2021). Posteriormente se ha producido un fuerte desarrollo en la academia brasileña asociado a un proceso político social bajo la denominación de gestão social en que se identifican temas comunes y conceptos, así como líneas divisorias bien marcadas (Peres \& Pereira, 2014) en la búsqueda de construir su propio campo (Cançado et al., 2015).

La gerencia social, a partir del concepto integrado propuesto por Aguiar-Barbosa y ChimMiki (2020) es definida como la gestión que, dada sus particularidades, se constituye en un modelo con característica propias, que se desenvuelve en forma participativa en espacios públicosprivados, teniendo como finalidad emancipar y desarrollar a los actores. En ese sentido, la 
autoridad decisoria es compartida entre los participantes de la acción (Chassot \& Allebrandt, 2015). En términos más afirmativos, la gerencia social es entendida como un proceso gerencial dialógico, deliberativo y participativo en el marco de la esfera pública que procura atender las necesidades de un sistema social, de una sociedad o, específicamente, de un territorio o región (Tenorio, 2005). Se desenvuelve en los ámbitos en que interaccionan el estado, el mercado y la sociedad civil.

Una de las formas de aproximarse a la producción científica sobre la gerencia social se da desde la bibliometría, la misma que permite lograr la medición de la difusión y el impacto de las publicaciones desde indicadores de producción de una entidad (investigador, revistas, grupo, institución), de desempeño (calidad de una revista, investigador o grupo) y estructurales (conexiones entre publicaciones, autores o campos de investigación) (Durieux \& Gevenois, 2010). En cuanto a los antecedentes sobre estudios bibliométricos referidos a la gerencia social, existen investigaciones con estrategias metodológicas cualitativas, cuantitativas y mixtas, como las desarrolladas por Iizuka et al. (2013), Peres y Pereira (2014), Da Silva et al. (2015), De Paula y Chim-Miki (2020), que tienen como unidades de análisis las revistas y artículos presentes en bases de datos y realizadas utilizando diferentes técnicas bibliométricas.

En base a lo expuesto anteriormente, la presente investigación pretende cubrir el vacío de conocimiento en torno a los avances de la actividad científica a partir de indicadores bibliométricos en el periodo que comprende desde los inicios de su concepción hasta los tiempos actuales. Para ello, se plantean los objetivos siguientes: i) analizar la tendencia evolutiva de la producción científica sobre gerencia social; ii) determinar los indicadores de producción según autores, países, categorías temáticas de Dimensions e idiomas, iii) identificar la red de coautoría entre autores, y iv) analizar el comportamiento de la evolución de tópicos de investigación en gerencia social.

\section{Metodología}

Este trabajo es un estudio cuantitativo de alcance descriptivo con metodología bibliométrica. Bajo la ecuación de búsqueda validada por los autores, se trabajó con la base datos Dimensions, que fue seleccionada dada la amplitud de referencias posibles de identificar a nivel mundial, esta misma ventaja es señalada en los estudios bibliométricos previos que usaron esta base de datos, pues ofrece una exhaustividad de documentos de revistas de todas las áreas del mundo (Khakimova et al., 2020; Rusydiana, 2021; Suharso et al., 2021).

Los descriptores de búsqueda para recuperar la información fueron en idioma español, portugués e inglés: "gestión social" OR "gerencia social” OR "gestão social" OR "social 
management" en los títulos o en el resumen. El criterio de inclusión fue que los documentos sean únicamente artículos científicos originales, desde el año 1989, fecha en que se publica el primer documento sobre el tema, hasta 2020. Al finalizar la recuperación de registros en octubre de 2020, se obtuvieron 1163. Luego de un proceso de exclusión quedaron 299 registros. La Figura I presenta la secuencia de obtención de los registros finales.

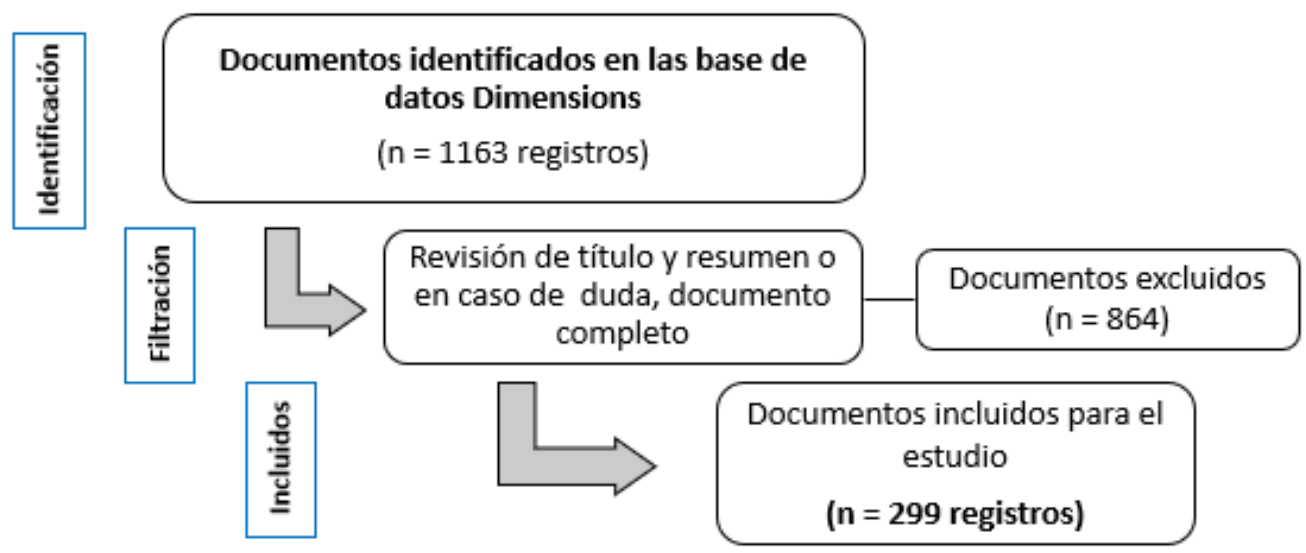

Figura I: Proceso metodológico de búsqueda, recuperación y selección de la información para el estudio

Para realizar el análisis de los datos se hizo uso de los programas Excel 2019, EndNote X7 y VOSviewer v1.6.15. Con los dos primeros se excluyeron y se prepararon los datos realizando un proceso de normalización de nombres de autores. No obstante, se empleó el modelo de Bradford, el cual se centra en la identificación de un núcleo básico de revistas sobre un tema determinado, de acuerdo con su productividad (Gorbea Portal, 1996), como se observa en la ecuación 1.

\section{(1) $p: p_{1}: p_{2}: 1: n: n^{2}$}

El programa VOSviewer se usó para mapear las redes bibliométricas basadas en distancia (van Eck \& Waltman, 2010; van Eck \& Waltman, 2014) según coautoría y coocurrencia de palabras clave. Una agrupación (clúster) es un conjunto de nodos o ítems estrechamente relacionados según el tipo de vínculo (unidad de medida) que se analiza, donde cada nodo es asignado exclusivamente a un clúster. Se usaron como unidades de análisis los autores y las palabras clave; y como vínculos o relaciones se emplearon: i) coautoría; y ii) coocurrencia de palabras clave, respectivamente. La 
red de coautoría de autores identifica la estructura social de la producción científica, es decir, la forma cómo colaboran los autores, reflejando a investigadores que trabajan en red y a los que trabajan de manera individual (Lazcano-Peña \& Reyes-Lillo, 2020); mientras que la red de coocurrencia de palabras clave sirve para identificar frentes de investigación que reflejan la estructura temática en el dominio de investigación (Van Eck \& Waltman, 2014; Fujita et al., 2014) de gerencia social.

\section{Resultados}

\section{Análisis de la tendencia evolutiva de la producción} científica sobre gerencia social

El término "gerencia social" se acuñó en 1989 y durante la primera década la producción de artículos era inferior a 11 trabajos. Sin embargo, el año 2019 se observa un incremento de documentos (Gráfico I). Asimismo, se observa un descenso considerable en 2020 y posiblemente son dos los factores que explican la baja producción: (1) la recopilación de datos fue obtenida en octubre de 2020; (2) la disminución de contenidos derivados de la emergencia sanitaria por el SARS-CoV-2.

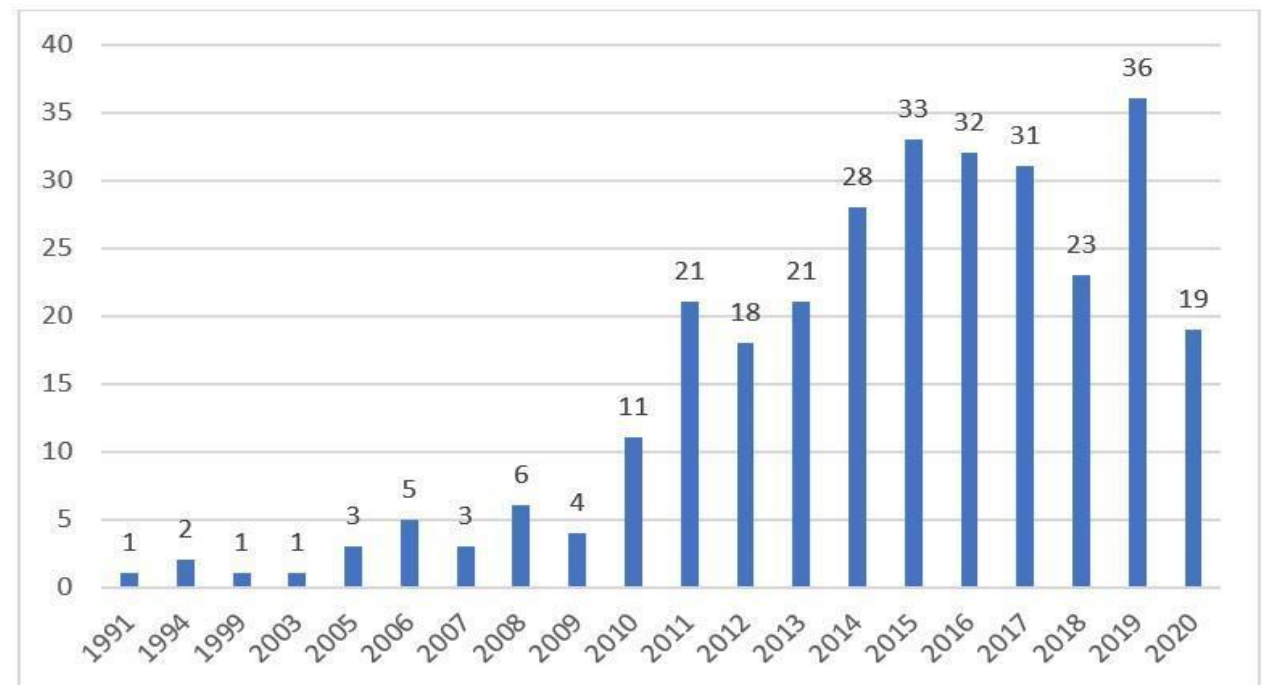

Gráfico I. Línea de tendencia de los artículos publicados por año sobre Gerencia Social. 


\section{Indicadores de producción según autores, países, categorías temáticas e idiomas en Dimensions}

Respecto al idioma, es interesante que el tema de la gerencia social se haya escrito principalmente en los idiomas portugués y español (Tabla 1). Aunque los documentos escritos en inglés se posicionan en tercer lugar, no representa un alto porcentaje ya que menos del 5\% de los trabajos está escrito en dicho idioma. Asimismo, sólo se encontraron cuatro artículos escritos en idioma ruso. Por su parte, el francés, vietnamita y ucraniano cuentan respectivamente con menos del $1 \%$ de la producción científica.

\section{Tabla 1}

Idioma en el que Dimensions dispone del mayor número de trabajos sobre gerencia social

\begin{tabular}{cccc}
\hline Posición & Idioma & ND & Porcentaje \\
\hline 1 & Portugués & 217 & $72.60 \%$ \\
2 & Español & 61 & $20.40 \%$ \\
3 & Inglés & 13 & $4.30 \%$ \\
4 & Ruso & 4 & $1.30 \%$ \\
5 & Francés & 2 & $0.70 \%$ \\
6 & Vietnamita & 1 & $0.30 \%$ \\
7 & Ucraniano & 1 & $0.30 \%$ \\
\hline
\end{tabular}

ND: Número de documentos

Entre los artículos examinados, se determinó la distribución geográfica de los autores de contacto gracias a que se incluía la dirección postal y adscripción en dichos artículos. Como se observa en la Tabla 2, el 74\% de los trabajos sobre gerencia social son de autores brasileños, mientras que solo un $20 \%$ de los autores son de varios países de habla hispana como Argentina, Chile, Colombia, Costa Rica, Cuba, Ecuador, España, México, Nicaragua, Panamá, Perú, Puerto Rico y Venezuela. También se encontró que el tema de gerencia social es de interés para canadienses, chinos, eslovacos, coreanos, noruegos, rusos, sudafricanos, ucranianos y vietnamitas. De forma general, los autores están distribuidos en los continentes de América, Europa, Asia y África. 
Tabla 2

Distribución geográfica de los autores de contacto

\begin{tabular}{|c|c|c|c|c|}
\hline Posición & País & ND & CGA & Porcentaje \\
\hline 1 & Brasil & 222 & 2828 & $74.20 \%$ \\
\hline 2 & Colombia & 21 & 98 & $7 \%$ \\
\hline 3 & Ecuador & 7 & 38 & $2.30 \%$ \\
\hline 4 & Chile & 6 & 138 & $2 \%$ \\
\hline 5 & Argentina & 5 & 111 & $1.70 \%$ \\
\hline 6 & Rusia & 5 & 0 & $1.70 \%$ \\
\hline 7 & España & 4 & 64 & $1.30 \%$ \\
\hline 8 & México & 4 & 29 & $1.30 \%$ \\
\hline 9 & Perú & 4 & 1 & $1.30 \%$ \\
\hline 10 & Venezuela & 4 & 2 & $1.30 \%$ \\
\hline 11 & China & 3 & 4 & $1 \%$ \\
\hline 12 & Canadá & 2 & 78 & $0.70 \%$ \\
\hline 13 & Nicaragua & 2 & 21 & $0.70 \%$ \\
\hline 14 & Costa Rica & 1 & 0 & $0.30 \%$ \\
\hline 15 & Cuba & 1 & 33 & $0.30 \%$ \\
\hline 16 & Eslovaquia & 1 & 0 & $0.30 \%$ \\
\hline 17 & Corea & 1 & 3 & $0.30 \%$ \\
\hline 18 & Noruega & 1 & 3 & $0.30 \%$ \\
\hline 19 & Panamá & 1 & 6 & $0.30 \%$ \\
\hline 20 & $\begin{array}{c}\text { Puerto } \\
\text { Rico }\end{array}$ & 1 & 0 & $0.30 \%$ \\
\hline 21 & Sudáfrica & 1 & 1 & $0.30 \%$ \\
\hline 22 & Ucrania & 1 & 0 & $0.30 \%$ \\
\hline 23 & Vietnam & 1 & 0 & $0.30 \%$ \\
\hline
\end{tabular}

ND: Número de documentos, CGA: Citas de Google Académico

*Nota: Citas obtenidas al 22 de diciembre de 2020.

\section{Análisis del comportamiento de la evolución de tópicos de investigación en gerencia social}

En la base de datos de Dimensions, el tema de gerencia social está orientado con un $58.9 \%$ en el área de Studies in Human Society, mientras el 21.4\% se clasifica en Commerce, Management, Tourism and Services (Tabla 3). El 5\% de los artículos pertenecen al área Economics y menos del 4\% se encuentra en Education, Environmental Science, Medical and Health Sciences, Psychology and Cognitive Sciences, Engineering, Information and Computing Sciences, History and Archaeology. Todas estas áreas, por una parte, muestran que podemos encontrar la gerencia social prioritariamente en estudios de la sociedad humana (Studies in Human Society), pues en ellos 
inciden sus aplicaciones y resultados, así como en una diversidad de materias, siendo de interés desde diferentes perspectivas teóricas, como se observa en la Tabla 4.

\section{Tabla 3}

Áreas del conocimiento donde Dimensions dispone del mayor número de trabajos sobre gerencia social (Top Ten)

\begin{tabular}{cccc}
\hline Posición & Materia & ND & Porcentaje \\
\hline 1 & Studies in Human Society & 176 & $58.90 \%$ \\
2 & Commerce, Management, Tourism and & 64 & $21.40 \%$ \\
3 & Services & 15 & $5 \%$ \\
4 & Economics & 12 & $4 \%$ \\
5 & Education & 11 & $3.70 \%$ \\
6 & Environmental Sciences & 6 & $2 \%$ \\
7 & Medical and Health Sciences & 3 & $1 \%$ \\
8 & Psychology and Cognitive Sciences & 3 & $1 \%$ \\
9 & Engineering & 3 & $1 \%$ \\
10 & Information and Computing Sciences & 2 & $0.70 \%$ \\
11 & History and Archaeology & 4 & $1.30 \%$ \\
\hline
\end{tabular}

ND: Número de documentos

\section{Tabla 4}

Subáreas del conocimiento donde Dimensions dispone del mayor número de trabajos sobre Gerencia social (Top Ten)

\begin{tabular}{cccc}
\hline Posición & Materia & ND & Porcentaje \\
\hline 1 & Sociology & 102 & $34.10 \%$ \\
2 & Business and Management & 62 & $20.70 \%$ \\
3 & Policy and Administration & 33 & $11 \%$ \\
4 & Political Science & 27 & $9 \%$ \\
5 & Applied Economics & 15 & $5 \%$ \\
6 & Human Geography & 13 & $4.30 \%$ \\
7 & Environmental Science and Management & 11 & $3.70 \%$ \\
8 & Specialist Studies In Education & 11 & $3.70 \%$ \\
9 & Public Health and Health Services & 6 & $2 \%$ \\
10 & Psychology & 3 & $1 \%$ \\
11 & Otras áreas (12) & 16 & $5.40 \%$ \\
\hline
\end{tabular}

ND: Número de documentos 
Sin duda, en la comunidad científica las revistas son un medio de comunicación destacado donde se difunden los resultados de investigación, tanto teóricos como prácticos. Como se observa en la Tabla 5, las primeras diez publicaciones son de origen brasileño, donde encontramos que la revista que concentra mayor número de artículos sobre Gerencia Social es Cadernos EBAPE BR, con 23 artículos, seguida de la revista denominada Desenvolvimento em Questão, con 19 trabajos. Es de destacar que existe actualmente una revista especializada en el tema de gerencia social y a su vez abarca una perspectiva interdisciplinar, que es la Revista Interdisciplinar de Gestão Social, con 14 artículos. También en la Tabla 5 se observa una dispersión de los artículos, lo que se explica porque alrededor de 144 revistas han publicado al menos un trabajo sobre gerencia social.

\section{Tabla 5}

Revistas registradas en Dimensions donde sobresale el número de trabajos sobre Gerencia Social (más de tres artículos)

\begin{tabular}{cccc}
\hline Posición & Revistas & ND & CGA \\
\hline 1 & Cadernos EBAPE BR & 23 & 529 \\
2 & Desenvolvimento em Questão & 19 & 106 \\
3 & Revista Interdisciplinar de Gestão Social & 14 & 14 \\
4 & Revista de Ciências da Administração & 14 & 87 \\
5 & NAU Social & 11 & 13 \\
6 & Administração Pública e Gestão Social & 10 & 18 \\
7 & Revista de Administração Pública & 10 & 431 \\
8 & Organizações \& Sociedade & 8 & 91 \\
9 & DRd - Desenvolvimento Regional em debate & 7 & 137 \\
10 & Cadernos Gestão Pública e Cidadania & 4 & 7 \\
11 & Revista de Políticas Públicas & 4 & 4 \\
12 & COLÓQUIO & 4 & 6 \\
13 & Revista do Serviço Público & 3 & 192 \\
14 & Revista de Gestão Social e Ambiental & 3 & 10 \\
15 & Serviço Social em Revista & 3 & 3 \\
16 & Sociedade Contabilidade e Gestão & 3 & 32 \\
17 & Raízes: Revista de Ciências Sociais e & 3 & 8 \\
18 & Econômicas & 156 & 1770 \\
\hline
\end{tabular}

ND: Número de documentos, CGA: Citas de Google Académico

*Nota: Citas obtenidas al 22 de diciembre de 2020. 
Por otra parte, se distinguen las tres zonas de acuerdo al modelo de Bradford: en la zona 1 se encuentra una distribución de seis revistas que concentran 91 artículos, mientras que la zona 2 se compone de 62 revistas con 115 artículos y la zona 3 contiene 93 títulos con 93 artículos (Gráfico II). Asimismo, la zona 1 alcanza una densidad de 15.1 artículos por revista, mientras la zona 3 tiene una densidad de 1 , lo que pone en evidencia el fenómeno de concentración y dispersión de la producción científica en cada una de dichas zonas. En el Gráfico II, en la parte observada se identifican dos curvas, en que la primera representa el núcleo de revistas más productivas, y aunque en parte las dos líneas se superponen, se ve las revistas de desempeño medio; y la curva que desciende nos muestra el inicio de la tercera zona, que incluye revistas con poca producción.

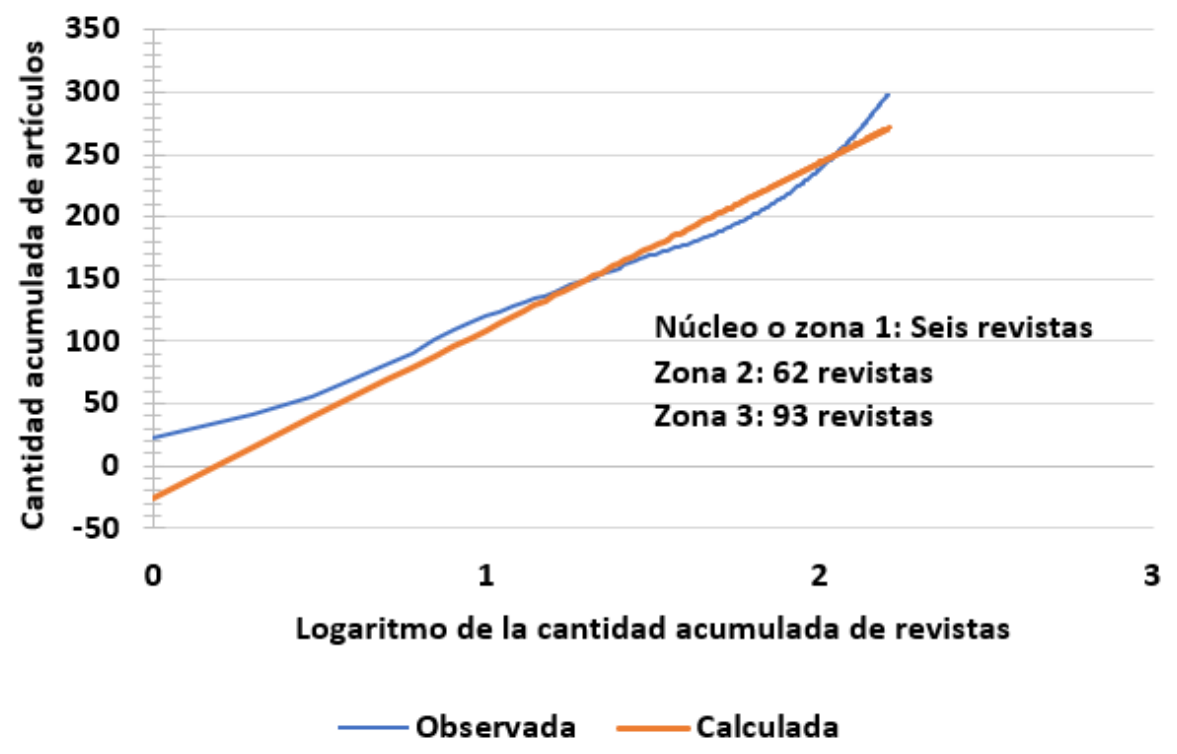

Gráfico II. Distribución en orden de productividad decreciente de artículos de revistas científicas (Modelo matemático de Bradford)

De los 299 artículos analizados se realizó un filtro para localizar los cinco artículos más citados en Google académico con el fin de encontrar aquellos trabajos que han contribuido de forma esencial en el desarrollo del tema de gerencia social (Tabla 6). Cabe destacar que el uso de Google Académico se debió por ser una herramienta libre además de proporcionar una cobertura mayor que Web of Science o Scopus (Martin-Martin et al., 2018) y proveer información de citas de revistas emergentes. Asimismo, se identifica que el trabajo más antiguo data del año 2002; y el más reciente, del año 2015. Por otro lado, tres artículos están firmados por un solo autor y dos están 
escritos en coautoría y pertenecen al mismo grupo de investigación interesado en el tema de la gerencia social.

\section{Tabla 6}

Los cinco artículos sobre gerencia social con mayor número de citas en Google Académico*

\begin{tabular}{cc}
\hline Artículos & CGA \\
\hline & \\
Paula, Ana Paula Paes de. (2005). Administração pública & \\
brasileira entre o gerencialismo e a gestão social. Revista de & 668 \\
Administração de Empresas, 45(1), 36-49. & \\
https://dx.doi.org/10.1590/S0034-75902005000100005 & \\
Cançado, Airton Cardoso, Tenório, Fernando Guilherme \& & 201 \\
Pereira, José Roberto. (2011). Gestão social: reflexões teóricas \\
e conceituais. Cadernos EBAPE.BR, 9(3), 681-703. \\
https://doi.org/10.1590/S1679-39512011000300002 \\
Mokate, Karen Marie. (2002). Convertendo o “monstro” em \\
aliado: a avaliação como ferramenta da gerência social. \\
Revista do Serviço Público, 53(1), 89-134. \\
$\begin{array}{l}\text { Lucchese, Patrícia T. R. (2003). Eqüidade na gestão } \\
\text { descentralizada do SUS: desafios para a redução de }\end{array}$ \\
desigualdades em saúde. Ciência \& Saúde Coletiva, 8(2), 439- \\
448. https://doi.org/10.1590/S1413-81232003000200009 \\
\\
Cançado, Airton Cardoso, Pereira, José Roberto, \& Tenório, \\
Fernando Guilherme. (2015). Fundamentos teóricos da gestão \\
social. DRd-Desenvolvimento Regional em debate, 5(1), 4-19.
\end{tabular}

CGA: Citas de Google Académico, Nota. Citas obtenidas al 22 de diciembre de 2020.

\section{Red de coautoría}

Respecto a la red de coautoría, se encontró que la mayoría de los artículos están escritos en colaboración. De los 299 artículos analizados, el $71.57 \%$ fueron firmados por entre dos a seis autores, es decir, el $1.67 \%$ de los trabajos tienen la firma de seis autores, el $3.67 \%$ de los artículos fue firmado por cinco autores; en lo restante, el 11.03\% fue registrado por cuatro autores, mientras el $24.74 \%$ de la producción fue escrita por tres autores; y la colaboración realizada por solo dos personas constituyó el $30.43 \%$ de los trabajos, lo cual significa que cerca de un tercio de los 
artículos fue hecho por dos autores, mientras el $28.42 \%$ es de autoría única. Cuando se analiza la variable del idioma de publicación y el lugar de adscripción del autor de contacto, se evidencia una alta correlación; se ve que una variable influye de manera importante en el comportamiento de la otra.

La Figura 2 presenta la red de coautoría, donde cada círculo (nodo) representa a un autor y su tamaño es directamente proporcional a la FTE de un autor con otros autores. De una población de 546 autores, se ha seleccionado aquellos que tienen al menos dos documentos, de los cuales 75 entran al umbral. El mapa muestra un total de 28 clústeres distintos representados cada uno por un color diferente. Esto muestra la diversidad de clústeres invisibles formados por la colaboración entre los investigadores de la temática abordada. En los clústeres rojo y verde se encuentra el mayor número de autores, con 10 integrantes en cada uno. Los colores distintos indican las diferentes agrupaciones de autores que están relacionados según la FTE de coautoría. Muchos autores relevantes se ubican en un lugar céntrico en sus respectivos clústeres, el cual resalta la importancia de sus estudios y la capacidad de relacionarse para publicar contribuciones sobre gerencia social.

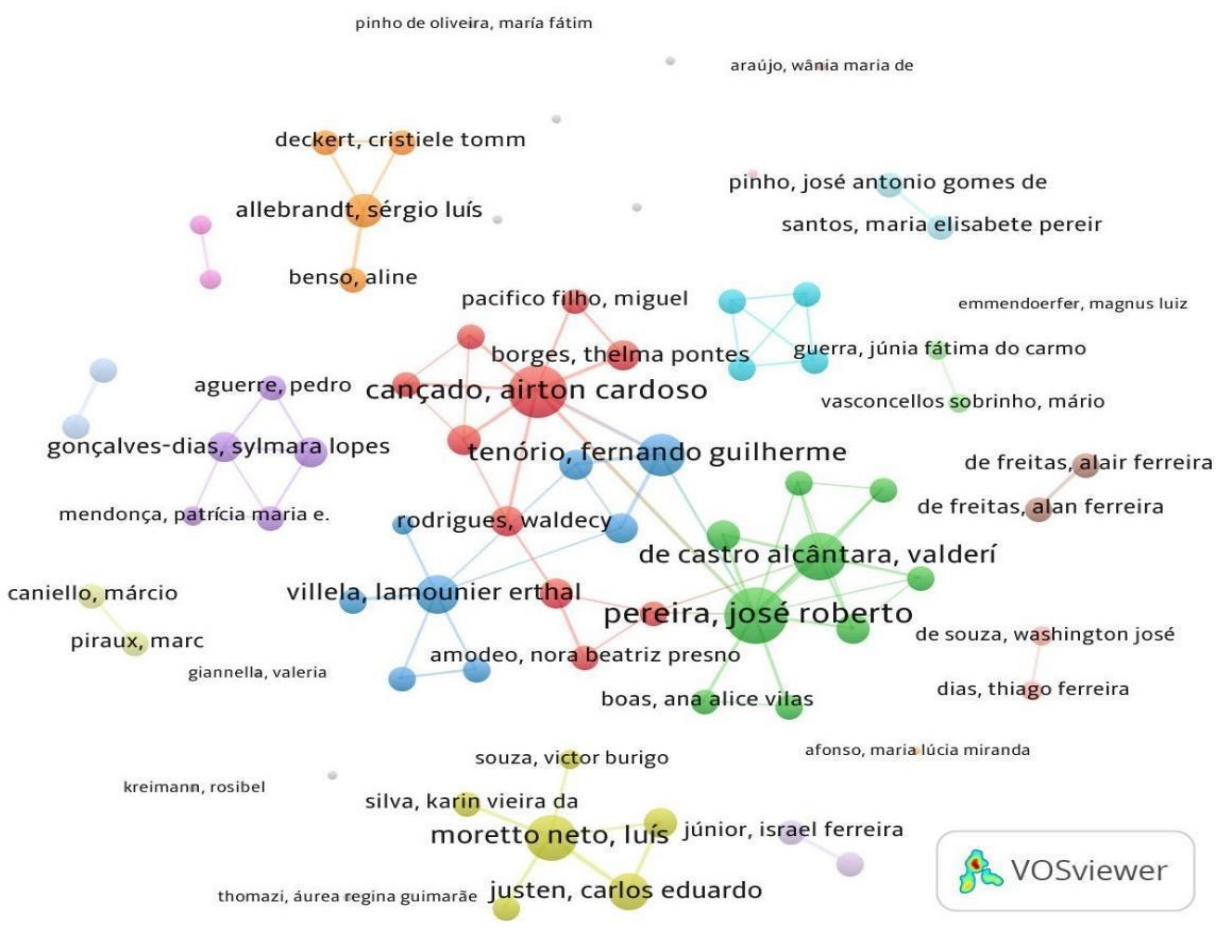

Figura II: Mapa de una red de coautoría entre autores 
En la Tabla 7 se presentan los ocho autores con mayor FTE. Pereira, José R. ocupa el primer lugar con una FTE de 15 y con 22 documentos, afiliado a la Universidad Federal de Lavras; le sigue Cançado, Airton Cardoso, con una FTE de 13 y con 17 documentos, afiliado a la Universidad Federal de Tocantins y así sucesivamente. Cabe resaltar que los ocho autores son brasileños, por lo que se puede concluir que hay un mayor esfuerzo de investigación por parte de la comunidad científica brasileña en el tema de gerencia social. Adicionalmente, la tabla muestra la información del perfil del autor, ya sea en Google Scholar o en Research Gate, lo que facilita la ubicación en caso surja una necesidad de contactarlos para realizar trabajos en colaboración o para algún evento científico.

Tabla 7

Los ocho autores con mayor FTE

\begin{tabular}{|c|c|c|c|c|}
\hline Autor & FTE & ND & País & $\begin{array}{c}\text { Perfil Google Académico o } \\
\text { ResearchGate }\end{array}$ \\
\hline $\begin{array}{l}\text { Pereira, José } \\
\text { Roberto }\end{array}$ & 15 & 22 & Brasil & $\begin{array}{l}\underline{\text { https://scholar.google.com/citations? }} \\
\underline{\text { user=0rICQsoAAAAJ\&hl=pt-PT }}\end{array}$ \\
\hline $\begin{array}{l}\text { Cançado, Airton } \\
\text { Cardoso }\end{array}$ & 13 & 17 & Brasil & $\begin{array}{l}\text { https://scholar.google.com/citations? } \\
\underline{\mathrm{hl}=\mathrm{pt}-\mathrm{PT} \& \text { user=2fB6Z3cAAAAJ }}\end{array}$ \\
\hline $\begin{array}{l}\text { De Castro Alcântara, } \\
\text { Valderí }\end{array}$ & 10 & 10 & Brasil & $\begin{array}{l}\text { https://scholar.google.com/citations? } \\
\underline{\text { hl=pt-PT\&user=RMBa9PYAAAAJ }}\end{array}$ \\
\hline Moretto Neto, Luís & 9 & 12 & Brasil & $\begin{array}{l}\text { https://scholar.google.com/citations? } \\
\underline{\mathrm{hl}=\mathrm{pt}-\mathrm{PT} \& \text { user=oPrxX4gAAAAJ }}\end{array}$ \\
\hline $\begin{array}{l}\text { Tenório, Fernando } \\
\text { Guilherme }\end{array}$ & 7 & 10 & Brasil & $\begin{array}{l}\text { https://scholar.google.com/citations? } \\
\underline{\mathrm{hl}=\mathrm{pt}-\mathrm{PT} \& \text { user=LfI-c-8AAAAJ }}\end{array}$ \\
\hline $\begin{array}{l}\text { Villela, Lamounier } \\
\text { Erthal }\end{array}$ & 6 & 8 & Brasil & $\begin{array}{l}\text { https://www.researchgate.net/profile } \\
\underline{\text { Lamounier_Villela }}\end{array}$ \\
\hline $\begin{array}{l}\text { Justen, Carlos } \\
\text { Eduardo }\end{array}$ & 5 & 6 & Brasil & $\begin{array}{l}\text { https://www.researchgate.net/scientif } \\
\text { ic-contributions/Carlos-Eduardo- } \\
\underline{\text { Justen-2048447532 }}\end{array}$ \\
\hline $\begin{array}{l}\text { Allebrandt, Sérgio } \\
\text { Luís }\end{array}$ & 4 & 6 & Brasil & $\begin{array}{l}\underline{\text { https://scholar.google.com/citations? }} \\
\text { hl=pt-PT\&user=Ji5g4aQAAAAJ }\end{array}$ \\
\hline
\end{tabular}


Nota: FTE: Fuerza total de los enlaces, ND: Número de documentos

\section{Red de coocurrencia de palabras clave}

La Figura 3 muestra la red de coocurrencia de las palabras clave cuya frecuencia de ocurrencia es de dos como mínimo. De las 424 palabras clave, 119 llegan al umbral. Cada nodo representa una palabra clave y su tamaño es proporcional a la FTE de una palabra con otras palabras clave. Es de esperar que social management es la palabra clave que tiene mayor FTE (144) y 104 enlaces con otras palabras clave. La red bibliométrica está formada por 10 clústeres representados cada uno por colores diferentes. Cada color agrupa palabras clave que están fuertemente relacionadas entre sí según la FTE de coocurrencia de palabras clave. De acuerdo a este vínculo, cuanto más cerca se encuentran dos palabras clave en la visualización, mayor será la relación entre ellas. Es decir, palabras clave que se ubican cerca la una de la otra tiende a aparecer en las mismas publicaciones; por ejemplo, democracy con state, o incubators con university education.

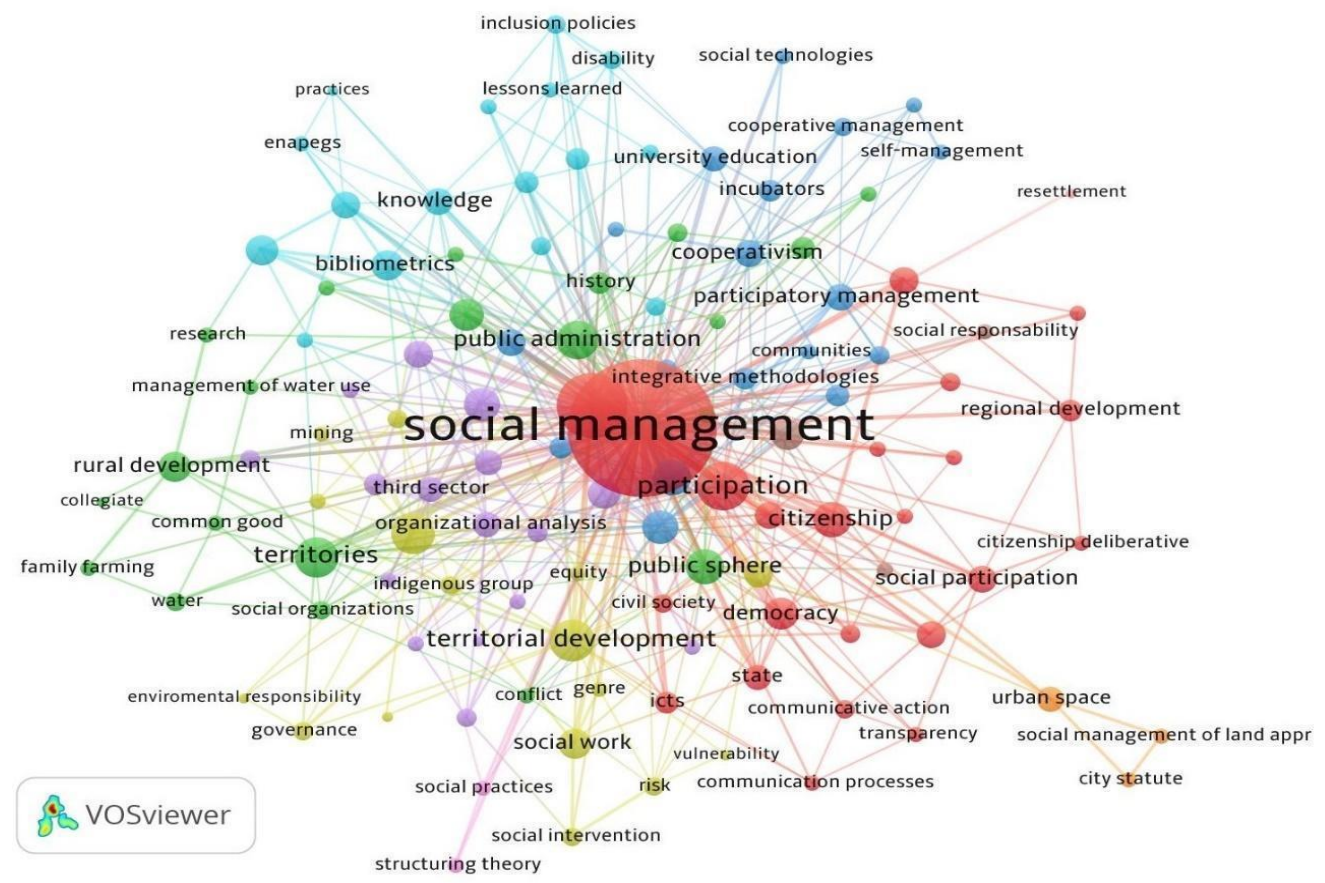

Figura III. Visualización de una red de coocurrencia de palabras clave Nota: Dos ocurrencias como mínimo. De las 406 palabras clave, 119 llegan al umbral. 
Para complementar el análisis, se presenta el mapa de superposición (overlay en inglés) (Figura IV) que permitió evidenciar la evolución de los tópicos más importantes dentro de este dominio de investigación según el año promedio de publicación de los documentos. Se visualizan en color violeta y celeste los ítems más frecuentes en artículos publicados en promedio los años 2012 y 2013, y en naranja y rojo los ítems que aparecen en los artículos publicados en promedio los años 2016 y 2017.

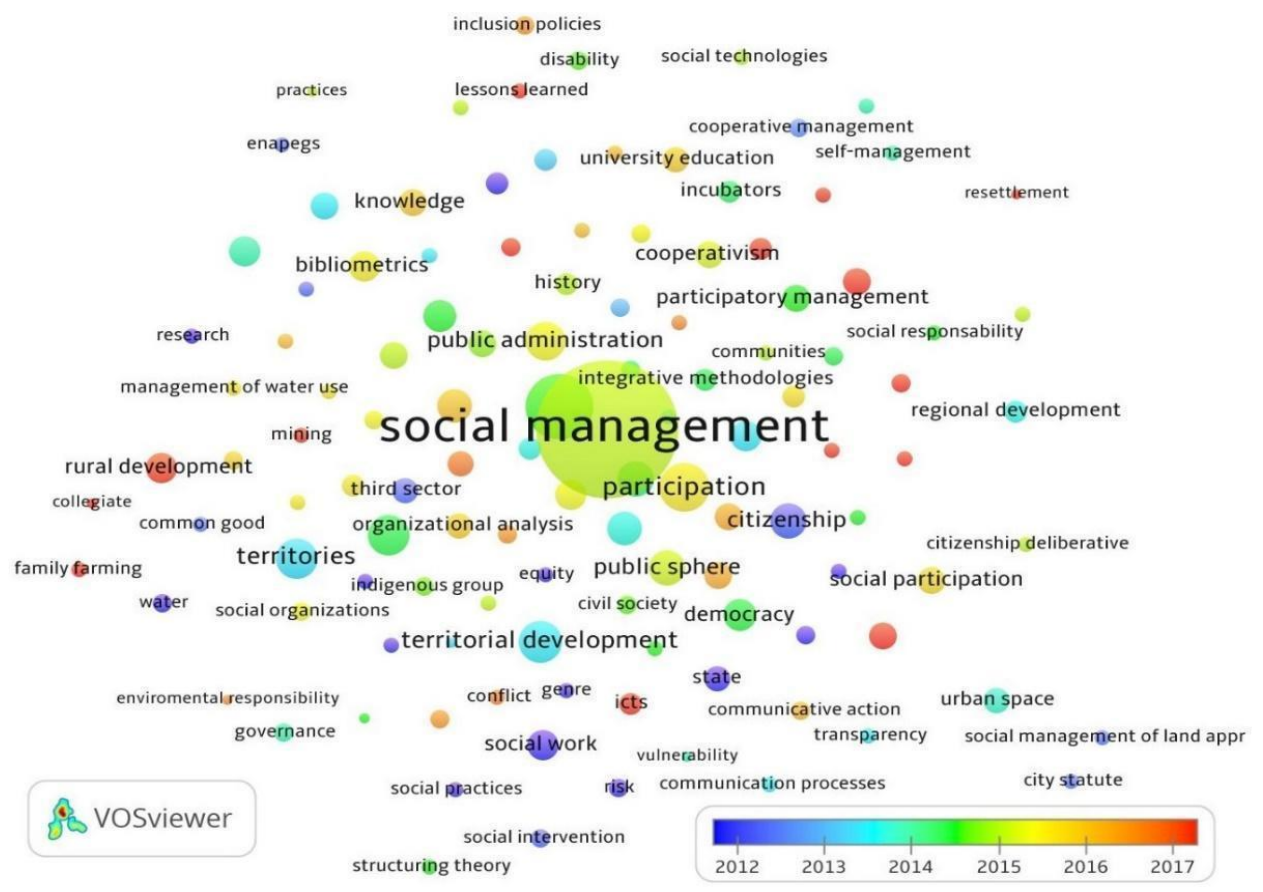

Figura IV. Visualización de un mapa de superposición de palabras clave

La Tabla 8 muestra la evolución de los tópicos de investigación por año promedio de publicación. Sin tomar en cuenta la palabra clave social management, por ser el tema central del estudio, se seleccionaron las 28 palabras clave más frecuentes en los documentos recuperados. Este análisis muestra las tendencias de investigación sobre social management de acuerdo a su ocurrencia y, por tanto, su importancia en el momento de desarrollo de la investigación; sin embargo, estas no excluyen otras temáticas de investigación. 


\section{Tabla 8}

Temáticas más frecuentes relacionadas con Gerencia Social por año promedio de publicación

\begin{tabular}{|c|c|c|}
\hline Palabra clave & Frecuencia & $\begin{array}{l}\text { Año promedio de } \\
\text { publicación }\end{array}$ \\
\hline Citizenship & 11 & \multirow{4}{*}{2012} \\
\hline Social work & 8 & \\
\hline State & 5 & \\
\hline Third sector & 5 & \\
\hline Territorial development & 14 & \multirow{5}{*}{2013} \\
\hline Territories & 13 & \\
\hline Solidary economy & 9 & \\
\hline Networking social & 6 & \\
\hline Strategic management & 4 & \\
\hline Sustainable development & 14 & \multirow{5}{*}{2014} \\
\hline Public management & 10 & \\
\hline Democracy & 8 & \\
\hline Urban space & 7 & \\
\hline Scientific production & 7 & \\
\hline Public politics & 35 & \multirow{5}{*}{2015} \\
\hline Participation & 19 & \\
\hline Public administration & 15 & \\
\hline Public sphere & 10 & \\
\hline Cooperativism & 6 & \\
\hline Management & 9 & \multirow{4}{*}{2016} \\
\hline Social participation & 6 & \\
\hline Knowledge & 6 & \\
\hline Deliberative citizenship & 6 & \\
\hline Rural development & 8 & 2017 \\
\hline
\end{tabular}


Jurgen Habermas

Interdisciplinary

Social control

Information and

Communication Technologies

(ICTs)

En relación a la temática que se presenta con mayor frecuencia en cada año podemos decir lo siguiente:

2012: el tema ciudadanía pone en discusión la actuación de diferentes actores en la gestión social como parte de un proceso de ciudadanía deliberativa; trabajo social es una disciplina muy importante relacionada a la gestión de las políticas públicas, las mismas que encuentran un nivel de concreción en los programas, proyectos y servicios

2013: el desarrollo territorial se torna en un fundamento importante para el desarrollo rural, en contraposición al enfoque vertical y centralizador. Asimismo, plantea estrategias a través del desarrollo técnico y la agroecología. Los trabajos se refieren a la gestión social en un territorio concreto para el desarrollo de una ciudadanía deliberativa y la construcción de poderes locales con mayor autonomía, en este sentido aparece el tema territorio. La temática referida a economía solidaria constituye una filosofía en que la economía está centrada en el ser humano antes que el lucro, valiéndose de la asociatividad, cooperación y autogestión para la emancipación de grupos socialmente relegados. En este aspecto se diferencias de los emprendimientos tradicionales

2014: el desarrollo sustentable, es un enfoque que pretende enfrentar las vulnerabilidades e impactos generados en las colectividades, por lo cual se plantean diversas iniciativas asentadas en un cambio radical como por ejemplo en el uso de los recursos no renovables. Gestión pública, referida al trabajo desplegado desde el estado para modernizar la esfera política, social y económica, y democracia, refiriéndose principalmente a procesos de la gestión social para lograr una participación más representativa en las instancias de intermediación pública

2015: la temática referida a políticas públicas refleja su carácter multidimensional y su aplicación en los diferentes niveles de la gestión del estado; participación como un factor clave para la generación de políticas públicas orientadas a la transformación social, y administración 
pública aparece como un tópico en el que se analizan aspectos teóricos y tecnologías modernas de gestión social.

2016: gerencia, son de particular importancia los estudios de las características y antecedentes de los modelos de gestión pública desde un enfoque gerencial.

2017: desarrollo rural, los científicos sociales encuentran diferentes enfoques de su dinámica y significado.

\section{Discusión}

Los resultados ponen en evidencia la tendencia creciente en cuanto a producción científica sobre la gerencia social a nivel mundial. La mayor producción corresponde a las publicaciones realizadas entre 2011 y 2019. Luego de un ligero descenso en el 2012, los años siguientes van en ascenso, del 2013 a 2015, observándose un aumento significativo en el 2019. Esta tendencia también es documentada por Iizuka et al. (2013) para el periodo 2007-2013 en la investigación realizada sobre la base de los artículos presentados en el ENAPEG en Brasil.

Es precisamente Brasil el país que presenta una posición de liderazgo en cuanto a la producción (74\%) para el periodo analizado. Sin embargo, esto no indica que otros países de Latinoamérica no se encuentren también interesados en la gerencia social como tema de investigación, pues como se presenta en la presente investigación existen publicaciones en español que corresponden a países de la región como Colombia, Ecuador, Chile con una producción mayor, seguido de México, Perú, Venezuela, Nicaragua, Costa Rica, Cuba, Panamá y Puerto Rico. A pesar de ser limitada la cantidad de fuentes bibliográficas para contrastar de forma específica los resultados de la presente investigación, es posible encontrar de forma general que la producción científica latinoamericana sobre administración, disciplina que alberga a la especialización de gerencia social en algunos países, se concentra principalmente en Brasil, Chile, México, Argentina y Colombia (Díaz-Contreras \& Ronda-Pupo, 2019).

Las revistas con mayores publicaciones fueron Cadernos EBAPE BR, seguida de la revista denominada Desenvolvimento em Questão. Es de destacar que existe actualmente una revista especializada en el tema de gerencia social y que a su vez abarca una perspectiva interdisciplinar, que es la Revista Interdisciplinar de Gestão Social. Las dos primeras han mantenido su productividad a lo largo de los años ya que también se destacan en la investigación realizada por Da Silva et al. (2015). Asimismo, los artículos más citados corresponden a los autores Paula (2005); 
Cançado et al. (2011); Mokate (2002); Lucchese (2003) y Cançado et al. (2015). El autor Cançado es referenciado como uno de los más citados en la base de datos Spell de acuerdo a los hallazgos obtenidos por Da Silva et al. (2015).

La presente investigación da cuenta que Brasil mantiene una alta productividad como resultado del debate permanente de la academia en relación con el tema, develando una significativa red de coautoría presente en 28 clusters distintos. En ese mismo sentido De Oliveira Menon y De Souza Coelho (2019) refieren que en los últimos 20 años la academia brasilera avanzó a partir del análisis de casos, elaboración de ensayos teóricos sobre el concepto y sus modelos desde una red de investigadores lo que le permite contar con una alta infraestructura, sustentada en programas de formación académica en universidades, grupos de investigación especializados, espacios de discusión y debate como los encuentros periódicos anuales, así como producción científica en revistas especializadas..

Los mapas de visualización de redes de coautoría y de coocurrencia de palabras clave dan cuenta de la estructura intelectual de la producción sobre la temática. En estos, se evidencia que Pereira y Cançado fueron los autores con mayor número de citas, y, junto con Moretto, fueron los autores con mayor fuerza total de enlace. Fuera del ámbito brasilero, la autora Mokate (2002), cuya publicación se ubica dentro de las perspectivas teóricas del inicio de la gerencia social junto con Kliksberg, B, es altamente citada en el periodo analizado. Para Peres y Pereira (2014) los conceptos que sustentan los enfoques surgidos alrededor de la gestión social provienen de la influencia de Boaventura Sousa Santos, Bernardo Kliksberg y Alberto Guerreiro Ramos.

La producción fue hecha en su mayoría en colaboración: el 71,6\% de las investigaciones fueron realizadas entre dos y seis autores, propiciando redes de conocimiento y de interés en tópicos concernientes a los aspectos epistemológicos, teóricos y prácticos de la gerencia social. Es evidente que cada vez es mayor la investigación colaborativa, tendencia que también se observa en el área de la administración como lo afirma Muñoz et al. (2017) al señalar que los artículos son producidos por dos o más compañeros.

La identificación de redes de coautoría y alianzas institucionales para el trabajo colaborativo mediante los mapas de visualización, se convierte en información valiosa para aunar esfuerzos en la profundización del conocimiento en países donde la temática sea relevante para los académicos y sus respectivas instituciones, tanto como para el propio país. En ese sentido, los resultados señalan que el mayor número de trabajos en relación a las áreas de conocimiento de la 
base de datos Dimensions, corresponden principalmente a: Studies in Human Society Commerce, Management, Tourism and Services Economics Education Environmental. Las temáticas más frecuentes relacionadas con la gerencia social según año fueron: Citizenship y Social work (2012), Territorial development y Territories (2013), Sustainable development y Public management (2014) Public politics y Participation (2015), Management y e Social participation (2016) y Rural development y Social control (2017). Algunas de estas tuvieron vigencia durante el periodo del 2007-2013 Territories, Networking social y Public management (Iizuka et al., 2013). Los resultados al respecto permitieron tener un panorama que hace posible identificar a los autores, publicaciones y las revistas que tuvieron preponderancia en la última década a partir del surgimiento de la gerencia social y que mantienen su presencia en el espectro académico.

La gerencia social, disciplina que no avanza sobre recetas o prescripciones (Boullosa, 2009) dada su naturaleza compleja y su carácter multidisciplinar presenta como desafío avanzar en la integración de enfoques desde los elementos teóricos como sobre las evidencias prácticas, para construir consensos hacia un paradigma (De Oliveira Menon \& De Souza Coelho, 2019). Este avance, a decir de estos autores, constituye la tercera generación en el campo de saber de la gestión social iniciado a partir del 2015.

Dado que el presente estudio se sustenta en una sola base de datos, se sugiere que estudios futuros aborden la temática en otras fuentes, tales como Scopus, Web of Science, Scielo, entre otras. De esta manera, se trabaje con un universo mayor de publicaciones y se logre una comprensión más amplia de este campo de conocimiento. Así mismo, trabajar únicamente con artículos científicos como unidades de análisis, excluyendo a libros que constituyen también una referencia de la producción, reduce el universo de información. Se considera como tarea pendiente para los investigadores una mayor socialización de sus hallazgos en publicaciones en revistas indexadas, entendido como uno de los medios principales para presentar resultados de investigaciones en el área.

\section{Conclusiones}

La presente investigación cumplió con los objetivos propuestos a partir de los indicadores bibliométricos de productividad y desempeño, a partir de los artículos científicos publicados en la base de datos Dimensions. Se evidencia la utilidad de la bibliometría como opción metodológica. En relación con las investigaciones bibliométricas anteriores, se puede señalar como contribuciones significativas la actualización de la productividad de autores por un periodo de 30 
años hasta el 2020, siendo que Da Silva et al. (2008), trabajaron este indicador del 2000 al 2005. Así mismo, se obtuvo como resultado 29 categorias temáticas relacionadas a la gerencia social, que son materia de abordaje desde la academia, mientras que Izuka et al. (2013) se concentraron en seis temáticas principales.

La tendencia creciente de la producción en Gerencia social revela la vigencia de esta disciplina. Se identificaron sus vínculos con otras áreas de conocimiento como Ciencias Sociales, Comercio, Administración, Política, Economía y Educación, brindando una perspectiva acerca de cuáles son los campos donde existe sinergia. Los resultados de esta investigación abren las puertas para seguir indagando sobre los diversos temas emergentes que aparecen con mayor frecuencia en los últimos años como: Desarrollo rural, Interdisciplinariedad, Control social, Tecnologías de Información y Comunicación en su relación con la Gerencia Social.

\section{Referencias}

Aguiar-Barbosa, A., y Chim-Miki, A. (2020). Evolução do conceito de gestão social (1990-2018): uma análise de copalavras. Cadernos Gestão Pública e Cidadania, 25 (80), 1-22. https://doi.org/10.12660/cgpc.v25n80.80525

Muñoz, L., Rodríguez Bolivar, M.P., y López-Hernández, A. (2017). Análisis bibliométrico sobre la implementación de las tecnologías de la información y la comunicación en las administraciones públicas: aportaciones y oportunidades de investigación. Innovar, 27 (63), 141-160. https://doi.org/10.15446/innovar.v26n63.60674

Araújo, E. T. (2014). Gestão Social. En R. de F. Boullosa, Dicionário para a formação em gestão social (pp. 85-90). CIAGS/UFBA. https://www.pucsp.br/sites/default/files/download/neats/Dicionario-para-a-Formacao-emgestao-social.pdf

Beaumont, M. (2016). Gestión social: estrategia y creación de valor. Pontificia Universidad Católica del Perú. http://repositorio.pucp.edu.pe/index/handle/123456789/54214

Boullosa, R. (2009). Contribuições conceituais e metodológicas para a avaliação de processos e práticas de gestão social a partir das perspectivas da policyanalysis e da abordagem social da aprendizagem. Colóquio Internacional sobre Poder Local. 11. CIAGS/UFBA.

Cançado, A., Rigo, A., y Pinheiro, L. (2016). Por una agenda de investigación para la gestión social: control social, paradigma, escala y cuadro de análisis. Praxis sociológica, 21, 6594. http://repositorio.ufba.br/ri/handle/ri/28350

Cançado, A., Tenorio, F., y Pereira, J. (2015). Fundamentos Teóricos da Gestão Social. DRd Desarrollo Regional en Debate, 5 (1), 4-19. https://doi.org/10.24302/drd.v5i1.703 
Cançado, A., Tenorio, F., y Pereyra, J. (2011). Gestión social: reflexiones teóricas y conceptuales. EBAPE.BR, 9 (3), 681-703. https://doi.org/10.1590/S1679-39512011000300002

Chassot, J. P., y Allebrandt, S. L. (2015). Gestión social de concejos municipales de desarrollo, $\begin{array}{llllll}\text { Rio Grande } & \text { Do } & \text { Sul. } & \text { LIDER, } & 17 & \text { (27), }\end{array}$ https://revistaliderchile.com/index.php/liderchile/article/view/58

Da Silva, F., Da Silva, C., Maciel, S., Buenos Aires, L., y Da Silva, C. (2015). A produção científica em gestão social no Brasil: uma análise a partir da Plataforma Spell.org. En I Encontro Nacional de ensino e pesquisa do campo de públicas (ENEPCP). https://conferencias.ufca.edu.br/index.php/enepcp/ENEPCP2015/paper/view/3928

De Oliveira Menon, I., y De Souza Coelho, F. (2019). Gestão social como campo do saber no brasil: uma investigação de sua produção científica pela modelagem de redes sociais (20052015). Cadernos de Gestão Pública e Cidadania, 24 (79), 1-27. https://doi.org/10.12660/cgpc.v24n79.79851

de Paula, A., y Chim-Miki, A. (2020). El surgimiento de la perspectiva de la competitividad turística social: Mapeo y clusterización de Redes Bibliométricas. Estudios y Perspectivas en Turismo, 29 (2), 390-405. https://dialnet.unirioja.es/servlet/articulo?codigo=7316507

Díaz-Contreras, C., y Ronda-Pupo, G. (2019). El impacto relativo de la producción científica latinoamericana sobre Administración. Interciencia, 44 (8), 454-460. https://www.redalyc.org/journal/339/33960960005/html/

Durieux, V., y Gevenois, P. A. (2010). Bibliometric indicators: quality measurements of scientific publication. Oak Brook, 255 (2), 342-351. https://doi.org/10.1148/radiol.09090626

Fujita, K., Kajikawa, Y., Mori, J., y Sakata, I. (2014). Detecting research fronts using different types of weighted citation networks. Journal of Engineering and Technology Management, 32, 129-146. https://doi.org/10.1016/j.jengtecman.2013.07.002

Gorbea Portal, S. (1996). El modelo matemático de Bradford: su aplicación a las revistas latinoamericanas de las ciencias bibliotecológica y de la información. Universidad Nacional Autónoma de México. https://ru.iibi.unam.mx/jspui/handle/IIBI_UNAM/L203

Iizuka, E., Antonio, L., y Junqueira, P. (2013). Produção acadêmica em Gestão Social: Visita aos anais dos enapegs de 2007 a 2012. Revista de Gestao Social e Ambiental, 7 (2), 71-85. https://doi.org/10.24857/RGSA.V7I2.796

INDES (2006). La gerencia social INDES. Documento de trabajo I-60. Departamento de Integración y Programas Regionales, Instituto Interamericano para el Desarrollo Social. https://publications.iadb.org/publications/spanish/document/La-Gerencia-SocialINDES.pdf

Khakimova, A., Zolotarev, O., y Berberova, M. (2020). Visualization of bibliometric networks of scientific publications on the study of the human factor in the operation of nuclear power 
plants based on the bibliographic database Dimensions. Scientific Visualization, 12 (2), 127 - 138. https://doi.org/10.26583/sv.12.2.10

Kliksberg, B. (1989). Gerencia social. Una revisión de la situación. En B. Kliksberg, ¿Cómo enfrentar la pobreza?. Grupo Editorial Latinoamericano.

Lazcano-Peña, D., y Reyes-Lilo, D. (2020). Redes académicas en la investigación en Comunicación en Chile: análisis de co-autorías en el trabajo científico. Revista Española de Documentación Científica, 43 (1), e259. https://doi.org/10.3989/redc.2020.1.1626

Maia, M. (2005). Gestão Social - Reconhecendo e construindo referenciais. Revista Virtual Textos y Contextos, 4 (1), 1-18. http://www.redalyc.org/articulo.oa?id=321527157020

Martin-Martin, A., Orduna-Malea, E., Thelwall, M., y Delgado López-Cózar, E. (2018). Google Scholar, Web of Science, and Scopus: A systematic comparison of citations in 252 subject $\begin{array}{lllll}\text { categories. Journal of Informetrics, } 12 & \text { (4), }\end{array}$ https://doi.org/10.1016/j.joi.2018.09.002

Mendonça, P., Gonçalves-Dias , S., y Junqueira , L. (2012). Gestão Social: notícias sobre o campo de estudos e práticas a partir da interações e debates do VI Enapegs. Revista de Administración Pública, $46 \quad$ (5), 1391-1408. https://doi.org/10.1590/S003476122012000500010

Oliveira-Ribeiro, R., Chim-Miki, A., y Marchado, P. de A. (2021). Assumptions of Social Management in the Brazilian Perspective: A Parallel with International Approaches. Brazilian Administration Review, 18 (1), 1-28. https://doi.org/10.1590/18077692 bar2021190110

Paula, A. P. (2005). Administração Pública Brasileira Entre o Gerencialismo e a Gestão Social. RAE-Revista de Administração de Empresas, 45 (1), 36-49. https://doi.org/10.1590/S003475902005000100005

Peres, M., y Pereira, J. (2014). Abordagens teóricas da Gestão Social: uma análise de citações exploratória. Cadernos EBAPE.BR, 12 (2), 221-236. https://doi.org/10.1590/167939519079

Real Academia Española (2014). Diccionario de la Real Academia Española, $23^{\circ}$ ed. Sitio web de Real Academia Española. httpss:// dle.rae.es

Rusydiana, A. (2021). Bibliometric analysis of journals, authors, and topics related to COVID-19 and Islamic finance listed in the Dimensions database by Biblioshiny. Science Editing, 8 (1), 72-78. https://doi.org/10.6087/kcse.232

Suharso, P., Setyowati, L., y Arifah, M. (2021). Bibliometric Analysis Related to Mathematical Research through Database Dimensions. Journal of Physics: Conference series, 176 (1). https://doi.org/10.1088/1742-6596/1776/1/012055

Sulbrant, J. (2002). Introducción a la Gerencia Social. INDES. 
Tenorio, F. (1997). Gestão social: uma Réplica. Revista do Mestrado em Administração e Desenvolvimento $\quad$ Empresarial, $\quad 13 \quad$ (2), http://revistaadmmade.estacio.br/index.php/admmade\%20/article/viewFile/37/29

Tenorio, F. (1998). Gestão social: uma perspectiva conceitual. Revista de Administración Pública, 32(5), 7-23. http://bibliotecadigital.fgv.br/ojs/index.php/rap/article/view/7754/6346

Tenorio, F. (2005). (Re)visitando o conceito de gestão social. Developement in Question, 3 (5), 101-124. https://doi.org/10.21527/2237-6453.2005.5.101-124

Tenorio, F., y Araújo, E. (2020). Una vez más, el concepto de gestión social. Cadernos EBAPE.BR, 18 (4), 891-905. https://doi.org/10.1590/1679-395120200105

Guilherme Tenorio, F., y Teixeira, M.A.C. (2021). O conceito de gestão social e a democracia regressiva no Brasil após 2016. Administração Pública e Gestão Social, 13 (2), 1-16. https://doi.org/10.21118/apgs.v13i2.10767

Van Eck, N., y Waltman, V. (2010). Software survey: VOSviewer, a computer program for bibliometric mapping. Scientometrics, 84 (2), 523-538. https://doi.org/10.1007/s11192009-0146-3

Van Eck, N., y Waltman, V. (2014). Visualizing Bibliometric Networks. En Y. Ding, R. Rousseau, y D. Wolfram, Measuring Scholarly Impact (pp. 285-320). Springer. https://doi.org/10.1007/978-3-319-10377-8_13

Van Eck, N., y Waltman, V. (2020). VOSViewer. (U. Leiden, Ed.) Manual de VOSViewer. Sitio web de VOSViewer. https://www.vosviewer.com/documentation/Manual_VOSviewer_1.6.17.pdf 